\title{
Risk factors for esophagojejunal anastomotic leakage in curative total gastrectomy combined with D2 lymph node dissection for gastric cancer: A retrospective cohort study
}

Jiadi Xing

Beijing Cancer Hospital

Maoxing Liu

Beijing Cancer Hospital

Xinyu Qi

Beijing Cancer Hospital

Jianhong Yu

Beijing Cancer Hospital

Yingcong Fan

Beijing Cancer Hospital

Kai Xu

Beijing Cancer Hospital

Pin Gao

Beijing Cancer Hospital

Fei Tan

Beijing Cancer Hospital

Zhendan Yao

Beijing Cancer Hospital

Nan Zhang

Beijing Cancer Hospital

Hong Yang

Beijing Cancer Hospital

Chenghai Zhang

Beijing Cancer Hospital

Ming Cui

Beijing Cancer Hospital

Xiangqian Su ( $\nabla$ suxiangqian@bjmu.edu.cn )

Beijing Cancer Hospital https://orcid.org/0000-0002-0667-8141 
Research article

Keywords: Esophagojejunal anastomotic leakage, Risk factors, Total gastrectomy

Posted Date: June 2nd, 2020

DOI: https://doi.org/10.21203/rs.3.rs-31924/v1

License: (c) (i) This work is licensed under a Creative Commons Attribution 4.0 International License. Read Full License 


\section{Abstract}

Background: This study aimed to explore the risk factors associated with esophagojejunal anastomotic leakage (EJAL) in curative total gastrectomy combined with $\mathrm{D} 2$ lymph node dissection for gastric cancer.

Methods: 390 consecutive patients receiving Roux-en-Y esophagojejunostomy reconstruction after surgery were reviewed. Multivariate analysis was performed using a logistic regression model to identify independent risk factors for EJAL.

Results: Of the 390 patients enrolled in this study, EJAL occurred in 10 patients (2.6\%). One patient (1/10) with EJAL died. Univariate analysis identified age $(P=0.025)$, alcohol consumption $(P=0.019)$, pulmonary insufficiency $(P=0.049)$, and intraoperative blood loss $(P=0.015)$ as risk factors for EJAL. Of these four risk factors, age $(P=0.043)$ and alcohol consumption $(P=0.043)$ were retained as independent risk factors by multivariate analysis.

Conclusions: Surgeons should be very careful about anastomotic leakage during the perioperative period, especially in patients with advanced age and a history of alcohol consumption. Pulmonary insufficiency and intraoperative blood loss, although not being identified as independent risk factors, should also be considered.

\section{Background}

Esophagojejunal anastomotic leakage (EJAL) is a common and serious postoperative complication of total gastrectomy. Moreover, EJAL has been identified as an independent negative prognostic factor for long-term survival [1]. The reported incidence of EJAL varies between $0.5 \%$ and $11.5 \%$ [1-9], and recently, Cetin DA [10] even reported an incidence of up to $16.2 \%$. These data highlight the requirement for the prevention of complications at the perioperative stage. Inconsistent reports on the risk factors of EJAL, long-term clinical data, maturity with the learning curve, and the now widespread use of mechanical stapling devices make previous research data gradually lose its relevance as reference material. To inform decision-making in gastric surgery, it is necessary to keep exploring the risk factors for EJAL.

Therefore, this study aimed to explore in detail the risk factors for EJAL in patients who had curative total gastrectomy combined with D2 lymph node dissection in our unit.

\section{Methods}

\section{Patients}

From April 2009 to April 2019, a total of 398 patients with gastric cancer received curative total gastrectomy combined with $\mathrm{D} 2$ lymph node dissection in our unit. Two patients who had duodenal stump leakage and a gastric stromal tumor, respectively, were excluded from the study. Two patients who had 
positive proximal margins, and four patients who had missing data were also excluded in this study; thus, 390 patients remained eligible for analysis.

\section{Surgical Technique}

The reconstruction method employed after total gastrectomy was the Roux-en-Y esophagojejunostomy. All esophagojejunal anastomoses were performed with a circular stapler. A suitably sized stapler for the esophageal and jejunal lumen was chosen at the surgeon's discretion. The integrity of the ring of the tissue that was retained by the circular stapler after accomplishment of the anastomosis was also examined. Finally, additional sutures were applied at large to reinforce the anastomosis.

\section{Definitions and variables}

The diagnosis of EJAL mainly relies on radiological and/or clinical findings. Contrast upper gastrointestinal angiography was routinely carried out for all patients after a median of six postoperative days in our unit, being feasible and low cost for patients. Radiological leakage was defined as a transudation outside of the lumen as seen on X-ray imaging as the patient was drinking the water-soluble contrast medium. Clinical leakage was defined as the leakage of intestinal fluid or turbid content from the surgical drain accompanied with fever, abdominal pain, and elevated leukocyte count, C-reactive protein (CRP), or procalcitonin (PCT). The definition of EJAL was basing on clinical leakage in present study. One patient with asymptomatic leakage that was only radiological diagnosed was not included in the EJAL group. The definition of an older person was made in accordance with the standards of the World Health Organization (WHO). Pulmonary insufficiency was defined as a forced expiratory volume measured for 1 s FEV1/Force Vital Capacity (FVC) $<0.70$ (obstructive lung disease) or Total Lung Capacity (TLC) $<80 \%$ (restrictive lung disease) [6]. Gastric cancer histopathological staging was performed according to the seventh edition of the International Union Against Cancer TNM classification [11]. "Alcohol consumption" was defined as alcohol intake as a limit of $1 \mathrm{U} /$ day for women and $2 \mathrm{U} /$ day $(1 \mathrm{U}$ of alcohol $=12 \mathrm{~g}$ of alcohol) for men, as stipulated in the Dietary Guidelines For Americans [12].

Patient-related, surgery-related, and tumor-related variables potentially associated with EJAL were recorded. Table 1 summarizes patient-related variables, including sex (female, male), age $(\leq 65,>65)$, smoking, alcohol consumption, hypertension, diabetes, body mass index (BMI) $\left(<25, \geq 25 \mathrm{~kg} / \mathrm{m}^{2}\right)$, neoadjuvant chemotherapy, pulmonary insufficiency, preoperative hemoglobin $(<90, \geq 90 \mathrm{~g} / \mathrm{L})$, preoperative serum albumin $(<35, \geq 35 \mathrm{~g} / \mathrm{L})$, and preoperative carcinoembryonic antigen $(<5, \geq 5 \mathrm{ng} / \mathrm{mL})$. Table 2 summarizes surgery-related variables, including operative approach (open, laparoscopic), duration of operation ( $<240, \geq 240 \mathrm{~min}$ ), intraoperative blood loss ( $<200, \geq 200 \mathrm{~mL}$ ), combined resection of other organs (spleen, pancreas, liver), and perioperative blood transfusion. Furthermore, Table 3 summarizes tumor-related variables, including tumor location (lower, middle, upper, whole), tumor size $(<4, \geq 4 \mathrm{~cm})$, lymph node dissection $(<16, \geq 16)$, pathology type of tumor (well, moderate, poor, other), depth of invasion (T0-4), and lymph node status (N0-3). In total, 23 potential risk factors were considered and analyzed in the present study. 


\section{EJAL Interventions}

The interventions for EJAL were as follows: (1) conservative treatment (with or without percutaneous drainage): fasting, antibiotics, nutritional support (enteral or parenteral), and insertion of a nasojejunal tube, and (2) surgical treatment: drainage, repair, or refashion of the anastomosis.

\section{Statistical Analyses}

Statistical analyses were performed using the SPSS version 19.0 (IBM Corp., Armonk, NY, USA). The continuous variables were dichotomized according to the clinical situation, standard values stipulated by state-of-the-art guidelines, or using the median value of each variable as the cutoff point. All patients were divided into two groups according to having experienced EJAL or not, and the groups were analyzed using the chi-squared test or Fisher's exact test. Variables with a probability $p$ value $<0.05$ in the univariate analysis and other factors that were thought to have important clinical significance were entered into the multivariate analysis. The multivariate analysis used a logistic regression model to investigate the risk factors associated with the incidence of EJAL, and a $p$ value $<0.05$ was considered statistically significant; odds ratios (OR) and their $95 \%$ confidence intervals $(\mathrm{Cl})$ were also provided.

\section{Results}

\section{Incidence of EJAL}

Of the 390 patients, EJAL was diagnosed in 10 (2.6\%). Among the ten EJAL patients, all patients were observed to have intestinal fluid or turbid content emerging from their drainage tube, nine had increased leukocyte levels, CRP, or PCT, eight patients developed fever, and four patients experienced abdominal pain. One patient $(1 / 10)$ died in the hospital due to septic shock caused by EJAL; the other nine eventually recovered by means of conservative treatment or surgery.

\section{Patient-related risk variables}

Table 1 displays the results of the univariate analysis of patient-related variables for EJAL. All the ten patients who developed EJAL were male, but this finding was not statistically significant $(P=0.073)$. There were no statistically significant differences in smoking, hypertension, diabetes, BMI, neoadjuvant chemotherapy, preoperative hemoglobin, preoperative serum albumin, or carcinoembryonic antigen between the two groups. EJAL was a more frequent manifestation in older patients $(P=0.025)$ and in patients who had preoperative pulmonary insufficiency $(P=0.049)$. Moreover, patients with history of excessive alcohol consumption were more likely to develop EJAL than those for whom no alcohol consumption had been reported $(P=0.019)$.

Table 1 Univariate analysis of patient-related variables for esophagojejunal anastomotic leakage 


\begin{tabular}{|c|c|c|c|c|}
\hline Variables & Leakage欧 & 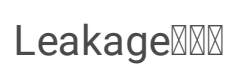 & $x^{2}$ & $P$ value \\
\hline Gender & & & 3.214 & 0.073 \\
\hline Male & 287 & 10 & & \\
\hline Female & 93 & 0 & & \\
\hline Age (years) & & & 5.014 & 0.025 \\
\hline$\leq 65$ & 275 & 4 & & \\
\hline$₫ 65$ & 105 & 6 & & \\
\hline Smoking & & & 0.096 & 0.757 \\
\hline No & 246 & 6 & & \\
\hline Yes & 134 & 4 & & \\
\hline Alcohol consumption & & & 5.473 & 0.019 \\
\hline No & 305 & 5 & & \\
\hline Yes & 75 & 5 & & \\
\hline Hypertension & & & 0.064 & 0.801 \\
\hline No & 291 & 8 & & \\
\hline Yes & 89 & 2 & & \\
\hline Diabetes & & & 1.076 & 0.300 \\
\hline No & 343 & 10 & & \\
\hline Yes & 37 & 0 & & \\
\hline $\mathrm{BMI}\left(\mathrm{kg} / \mathrm{m}^{2}\right)$ & & & 1.838 & 0.175 \\
\hline$\bowtie 25$ & 266 & 5 & & \\
\hline$\geq 25$ & 114 & 5 & & \\
\hline Neoadjuvant chemotherapy & & & 0.641 & 0.423 \\
\hline No & 303 & 9 & & \\
\hline Yes & 77 & 1 & & \\
\hline Pulmonary insufficiency & & & 3.866 & 0.049 \\
\hline No & 292 & 5 & & \\
\hline Yes & 88 & 5 & & \\
\hline 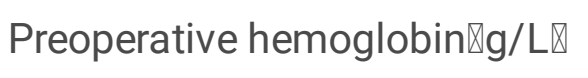 & & & 0.584 & 0.445 \\
\hline
\end{tabular}




\begin{tabular}{|c|c|c|c|c|}
\hline$\triangle 90$ & 21 & 0 & & \\
\hline$\geq 90$ & 359 & 10 & & \\
\hline Preoperative serum albumin (g/L) & & & 2.467 & 0.116 \\
\hline$\nabla 35$ & 49 & 3 & & \\
\hline$\geq 35$ & 331 & 7 & & \\
\hline Preoperative carcinoembryonic antigen $(\mathrm{ng} / \mathrm{ml})$ & & & 0.055 & 0.815 \\
\hline$\nabla 5$ & 292 & 8 & & \\
\hline$\geq 5$ & 88 & 2 & & \\
\hline
\end{tabular}

Abbreviation: BMI Body mass index; $\chi 2$ chi-square test; There were statistically significant differences for data in Italics $(p<0.05)$

\section{Surgery-related variables}

Table 2 shows the results of the univariate analysis of surgery-related variables for EJAL. Operational approach, duration of operation, combined resection with other organs (spleen, pancreas, liver), and perioperative blood transfusion were not statistically significant risk factors for EJAL. However, patients who had suffered extensive intraoperative blood loss were more likely to develop EJAL than those who had not $(P=0.015)$.

Table 2 Univariate analysis of surgery-related variables for esophagojejunal anastomotic leakage 


\begin{tabular}{|c|c|c|c|c|}
\hline Variables & Leakage欧 & Leakage怄 & $x^{2}$ & $P$ value \\
\hline Operative approach & & & 0.183 & 0.669 \\
\hline Open & 178 & 4 & & \\
\hline Laparoscopic & 202 & 6 & & \\
\hline Duration of operation $\rrbracket \min \rrbracket$ & & & 1.441 & 0.230 \\
\hline$\varangle 240$ & 147 & 2 & & \\
\hline$\geq 240$ & 233 & 8 & & \\
\hline Blood loss $\triangle \mathrm{ml} \mathbb{Z}$ & & & 5.885 & 0.015 \\
\hline$\varangle 200$ & 330 & 6 & & \\
\hline$\geq 200$ & 50 & 4 & & \\
\hline Combined organ resection & & & 0.242 & 0.622 \\
\hline No & 371 & 10 & & \\
\hline Yes & 9 & 0 & & \\
\hline Perioperative blood transfusion & & & 0.063 & 0.802 \\
\hline No & 331 & 9 & & \\
\hline Yes & 48 & 2 & & \\
\hline
\end{tabular}

Abbreviation: $\chi 2$ chi-square test; There were statistically significant differences for data in Italics $(\mathrm{p}<$ 0.05)

\section{Tumor-related variables}

Table 3 lists the results with regard to the univariate analysis of tumor-related variables for EJAL. The location of the tumor, tumor size, lymph node dissection, pathology type of tumor, depth of invasion, and lymph node status were not risk factors significantly associated with EJAL.

The multivariate analysis revealed that age $(P=0.043$, OR 3.882 [95\% $\mathrm{Cl} 1.045-14.422])$ and alcohol consumption ( $P=0.043$, OR 3.828 [95\% Cl 1.043-14.050]) were independent risk factors of EJAL (Table 4).

Table 3 Univariate analysis of tumor-related variables for esophagojejunal anastomotic leakage 


\begin{tabular}{|c|c|c|c|c|}
\hline Variables & Leakage怄 & Leakage欧 & $x^{2}$ & $P$ value \\
\hline Location of tumor & & & 1.761 & 0.623 \\
\hline Lower & 23 & 0 & & \\
\hline Middle & 94 & 3 & & \\
\hline Upper & 229 & 7 & & \\
\hline Whole & 34 & 0 & & \\
\hline Size of tumor $\ \mathrm{~cm} \rrbracket$ & & & 0.033 & 0.855 \\
\hline$\otimes 4$ & 163 & 4 & & \\
\hline$\geq 4$ & 217 & 6 & & \\
\hline Lymph node dissection & & & 0.497 & 0.481 \\
\hline Q16 & 18 & 0 & & \\
\hline$\geq 16$ & 362 & 10 & & \\
\hline Pathology type of tumor & & & 2.768 & 0.429 \\
\hline Well & 10 & 0 & & \\
\hline Moderate & 97 & 2 & & \\
\hline Poor & 247 & 6 & & \\
\hline Other & 26 & 2 & & \\
\hline Depth of invasion & & & 1.395 & 0.845 \\
\hline T0 & 3 & 0 & & \\
\hline T1 & 45 & 1 & & \\
\hline $\mathrm{T} 2$ & 41 & 2 & & \\
\hline T3 & 168 & 5 & & \\
\hline T4 & 123 & 2 & & \\
\hline Lymph node status & & & 4.492 & 0.481 \\
\hline NO & 140 & 5 & & \\
\hline N1 & 67 & 0 & & \\
\hline N2 & 83 & 4 & & \\
\hline N3 & 90 & 1 & & \\
\hline
\end{tabular}


Abbreviation: $\chi 2$ chi-square test; There were statistically significant differences for data in Italics $(\mathrm{p}<$ 0.05)

Table 4 Multivariate analysis of the risk factors for esophagojejunal anastomotic leakage

\begin{tabular}{llll} 
Variables & $P$ value & Odds ratio & $95 \% \mathrm{Cl}$ \\
\hline Age (years) & 0.043 & 3.882 & $1.045-14.422$ \\
\hline Alcohol consumption & 0.043 & 3.828 & $1.043-14.050$
\end{tabular}

Abbreviation: There were statistically significant differences for data in Italics $(p<0.05)$

\section{Discussion}

EJAL is a serious and potentially fatal complication after gastric surgery. It has been reported that EJAL has a mortality rate of up to $50 \%$ and is the major reason of postoperative death after surgery [13]. The present study found the incidence of EJAL was 2.6\% (10/390), which was similar to a recent quality meta-analysis with 2484 gastric cancer patients that reported the incidence of EJAL after total gastrectomy was $2.5 \%$ [14]. Moreover, this study also found a mortality rate of $10 \%(1 / 10)$, which further highlights why the risk of developing EJAL should be evaluated during the perioperative period.

Identification of risk factors of EJAL helps reduce the incidence of this condition in the clinic. Previously reported risk factors have mainly included patient-, surgery-, and tumor-related factors. In the present study, the univariate analysis revealed that age $>65$ years, alcohol consumption, pulmonary insufficiency, and intraoperative blood loss were risk factors associated with EJAL. The multivariate analysis demonstrated that age $>65$ years and alcohol consumption were the independent risk factors associated with EJAL in gastric surgery.

The present study also found that EJAL was more likely to occur in patients of advanced age, which is in line with two previous studies [6,9]. Gastric cancer occurs more frequently in older patients.

Unfortunately, older patients tend to be in poorer physical and wound healing condition and often suffer from malnutrition and comorbidities, making it more difficult for them to withstand the stress of surgery and keep physiological functions in balance, thereby to a certain degree increasing the risk of anastomotic leakage. Due to the poor ability of older patients to respond to stimuli, the early clinical symptoms of anastomotic leakage might be atypical and prone to be missed or misdiagnosed; thus, more attention should be paid to EJAL in older patients. Moreover, it was worth noting that it is still a matter of controversy whether diabetes affects the incidence of anastomotic leakage. Diabetes is well known to affect wound healing, not only including surgical incisions [15] but also intestinal anastomoses [16]. Kazuhiro [7] reported that poor preoperative diabetic control was an independent risk factor for EJAL. However, all ten patients who developed EJAL in this study did not have diabetes, and several previous 
reports $[1,3,5-6,9-10]$ also failed to find any association between diabetes and EJAL till this moment. Therefore, this issue deserve further discussion in the future.

Although Isozaki [3] and Sauvanet [17] successively reported that pulmonary insufficiency was not a risk factor for EJAL, Wu [18] found that respiratory disease was associated with postoperative complications after gastric surgery. The present study revealed that pulmonary insufficiency was associated with EJAL, but not an independent risk factor of EJAL. This may be explained by poor oxygen supply due to pulmonary insufficiency, combined with restricted breathing due to the pain after abdominal surgery, both of which may affect the healing process after gastric surgery. Moreover, the anastomotic leakage could aggravate impaired lung infection, and a vicious circle could develop. A high-quality randomized controlled trial by Schietroma [19] found that the risk of EJAL was $49 \%$ lower in patients who received $80 \% \mathrm{FiO} 2$ than in those who received $30 \% \mathrm{FiO} 2$ during and $6 \mathrm{~h}$ after open total gastrectomy surgery; this issue deserves further attention.

Although anastomotic leakage might be a complication driven by many factors, alcohol consumption has previously been associated with increased postoperative complications in colorectal cancer patients [20-21]. Rullier and Sorensen [22-23] reported that smoking and alcohol abuse were major risk factors for anastomotic leakage in colorectal surgery. Thomas [24] further demonstrated that an alcohol intake of more than $60 \mathrm{~g} /$ day was associated with an increased risk of anastomotic leakage in colorectal surgery. However, such analyses have rarely been published in studies of gastric cancer. To the best of our knowledge, this is the first study to report alcohol abuse being associated with EJAL upon gastric surgery. Alcoholism may affect the healing process and lead to impaired anastomotic integrity in various ways described below. Alcohol has been recognized as an influential factor in hemostasis, and excessive alcohol consumption may lead to increased perioperative bleeding because of bone marrow toxicity and decreased levels of fibrinogen, VII factor, and von Willebrand factor [25-27]. Cardiac insufficiency, immunosuppression, and hemostasis have also been demonstrated in symptom-free alcohol abusers with postoperative complications [28]. Therefore, preoperative use of alcohol should be identified and managed appropriately before surgical operation. Moreover, a history of alcohol consumption is an influential factor in the development of postoperative complications that clinicians should not forget. To obtain beneficial short-term reversibility of physiological impairments, 4-week alcohol abstinence prior to surgery has been advised [21]. Several studies [20-24] also revealed that smoking was a risk factor for anastomotic leakage and increased the postoperative complications upon rectal surgery. Although $40 \%$ of patients $(4 / 10)$ with a history of smoking developed EJAL, this factor failed to reach statistical significance in the present study but should be further discussed in the future.

With the arrival of the "minimally invasive" era, a significant reduction of intraoperative bleeding becomes possible. Though intraoperative blood loss was not an independent risk factor in this study, the authors speculate that it should not be ignored in gastric surgery. On the one hand, extensive blood loss during operation might impair the blood supply around the anastomosis, resulting in insufficient blood supply around the anastomosis and further increasing the risk of anastomotic leakage. One the other hand, the decrease in hemoglobin resulting from extensive blood loss will weaken the oxygen-carrying capacity, 
thereby causing anastomotic leakage. Three previous studies $[1,6,10]$ reported that longer duration of operation and combined organ resection including splenectomy or pancreatectomy were associated with anastomotic leakage, increasing the risk of morbidity. However, the present study failed to reach this conclusion, and the main reason for this may lie in staff experience and the use of optimized mechanical devices, which make the surgery faster and more secure, ultimately decreasing the incidence of anastomotic leakage. In recent years, neoadjuvant chemotherapy has been used more frequently in the treatment of advanced gastric cancer. However, only one patient (1/78) who received neoadjuvant chemotherapy developed EJAL after surgery and did not achieve significance, which was consistent with the study of Deguchi [6]. Therefore, it might not be a risk factor of EJAL.

One of the most important findings of this study was the identification of alcohol consumption being an independent risk factor for EJAL in patients receiving surgery for gastric cancer. However, several limitations should also be considered. First, the incidence of EJAL was much lower (2.6\%) than most previous studies which might hinder the discovery of more risk factors of EJAL in this study. Second, selection bias of patients and difficulty in collection data were also inevitable due to its retrospective nature. Third, no survival analysis was performed due to inadequate 5-year follow-up data available for analysis, which was also one deficiency in this study. However, research involving survival analysis is ongoing in our unit and will also be reported in the future.

\section{Conclusion}

In conclusion, although postoperative anastomotic leakage has a high mortality rate, it is controllable. During the perioperative period, surgeons should pay attention to the risk of anastomotic leakage after gastric surgery, especially in older patients ( $>65$ years old) and in patients with a history of alcohol consumption $>2$ units/day. Pulmonary insufficiency and intraoperative blood loss ( $\geq 200 \mathrm{ml}$ ), although not identified as independent risk factors in the present study, also deserved attention in this regard.

\section{Abbreviations}

EJAL: Esophagojejunal anastomotic leakage; CRP: C-reactive protein; PCT: Procalcitonin; WHO: World health organization; BMI: Body mass index; RCT: Randomized controlled trial

\section{Declarations}

\section{Ethics approval and consent to participate}

This study complied with the ethical guidelines of the Declaration of Helsinki and was approved by the Medical Ethics Committee of Peking University Cancer Hospital.

\section{Consent for publication}

Consent for publication was obtained from each patient included in this study. 
Availability of data and materials

The datasets used and analyzed during the current study are available from the corresponding author Xiangqian Su

\section{Competing interests}

All authors declare that there is no conflict of interest.

\section{Funding}

This study was funded by the grants from National Natural Science Foundation of China (81672439, 81450028, 81272766, 81470129, 81402346),Beijing Natural Science Foundation (7162039, 7132054, 7172042), Beijing Health System High-level Health Technical Personnel Training Program (20153-074), Capitals Funds for Health Improvement and Research (CFH 20182-2153), the Beijing Municipal Administration of Hospitals Clinical Medicine Development of Special Funding Support (XM201309, ZYLX201701)

\section{Acknowledgements}

Not applicable.

\section{Authors' contributions}

XQS contributed to the study conception and design. XYQ contributed to acquisition of data. JHY, YCF, $\mathrm{KX}, \mathrm{PG}$ and FT contributed to analysis and interpretation of data. ZDY, NZ, HY, CHZ and MC contributed to critical revision. JDX and MXL contributed to drafting of manuscript. All authors approved the final version of this manuscript.

\section{References}

1. Sierzega M, Kolodziejczyk P, Kulig J, et al. Impact of anastomotic leakage on long-term survival after total gastrectomy for carcinoma of the stomach. Br J Surg. 2010;97(7): 1035-42.

2. Hyodo M, Hosoya Y, Hirashima Y, et al. Minimum leakage rate $(0.5 \%)$ of stapled esophagojejunostomy with sacrifice of a small part of the jejunum after total gastrectomy in 390 consecutive patients. Dig Surg. 2007;24(3): 169-72.

3. Isozaki H, Okajima K, Ichinona T, et al. Risk factors of esophagojejunal anastomotic leakage after total gastrectomy for gastric cancer. Hepatogastroenterology. 1997;44(17):1509-12.

4. Ichikawa $D$, Kurioka $H$, Yamaguchi T, et al. Postoperative complications following gastrectomy for gastric cancer during the last decade. Hepatogastroenterology. 2004;51(56):613-17.

5. Meyer L, Meyer F, Dralle H, et al. Insufficiency risk of esophagojejunal anastomosis after total abdominal gastrectomy for gastric carcinoma. Langenbecks Arch Surg. 2005;390(6):510-16. 
6. Deguchi Y, Fukagawa T, Morita S, et al. Identification of Risk Factors for Esophagojejunal Anastomotic Leakage after Gastric Surgery. World J Surg. 2012;36(7):1617-22.

7. Migita K, Takayama T, Matsumoto S, et al. Risk factors for esophagojejunal anastomotic leakage after elective gastrectomy for gastric cancer. J Gastrointest Surg. 2012;16(9):1659-65.

8. Zilling T, Olseen P, Walther BS. Prediction of hospital stay after total gastrectomy. Anticancer Res. 1997;17(2B):1355-59.

9. Tu RH, Lin JX, Zheng $\mathrm{CH}$, et al. Development of a nomogram for predicting the risk of anastomotic leakage after a gastrectomy for gastric cancer. Eur J Surg Oncol. 2017;43(2) 485-92.

10. Cetin DA, Gündeş E, Ciyiltepe $H$, et al. A. Risk factors and laboratory markers used to predict leakage in esophagojejunal anastomotic leakage after total gastrectomy. Turk J Surg. 2019;35(1):6-12.

11. Sobin LH, Gospodarowicz MK, Wittekind C. International Union Against Cancer (UICC). TNM classification of malignant tumours. 7th ed. Wiley-Blackwell, New York, 2010.

12. S. Department of Agriculture and U.S. Department of Health and Human Services. In: Dietary Guidelines for Americans, chapter 3. 2012.

13. Makuuchi R, Irino T, Tanizawa Y, et al. Esophagojejunal anastomotic leakage following gastrectomy for gastric cancer. Surgery Today. (2019) 49(3):187-96.

14. Inokuchi M, Otsuki S, Fujimori Y, et al. Systematic review of anastomotic complications of esophagojejunostomy after laparoscopic total gastrectomy. World J Gastroenterol. 2015; 21(32):9656-65.

15. Black E, Vibe-Petersen J, Jorgensen $L N$, et al. Decrease of collagen deposition in wound repair in type 1 diabetes independent of glycemic control. Arch Surg 2003;138(1):34-40.

16. Onodera $H$, Ikeuchi $D$, Nagayama S, Imamura M. Weakness of anastomotic site in diabetic rats is caused by changes in the integrity of newly formed collagen. Dig Surg. 2004;21(2):146-51.

17. Sauvanet A, Mariette $C$, Thomas $P$, et al. Mortality and morbidity after resection for adenocarcinoma of the gastroesophageal junction: predictive factors. J Am Coll Surg. 2005;201(2):253-62.

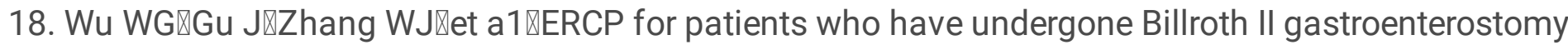
and Braun anastomosis $₫$ World J Gastroenterol $₫ 2014 \llbracket 20$ (2) 607-10.

19. Schietroma M, Cecilia EM, Carlei F, et al. Prevention of anastomotic leakage after total gastrectomy with perioperative supplemental oxygen administration: a prospective randomized, double-blind, controlled single-center trial. Ann Surg Oncol. 2013;20(5):1584-90.

20. van Rooijen S, Carli F, Dalton SO, et al. Preoperative modifiable risk factors in colorectal surgery: an observational cohort study identifying the possible value of prehabilitation. Acta Oncol. 2017;56(2):329-34.

21. Kennedy ND, Winter DC. Impact of alcohol \& smoking on the surgical management of gastrointestinal patients. Best Pract Res Clin Gastroenterol. 2017; 31(5): 589-95.

22. Rullier $\mathrm{E}$, Laurent $\mathrm{C}$, Garrelon JL, et al. Risk factors for anastomotic leakage after resection of rectal cancer. Br J Surg.1998; 85(3):355-58 
23. Sorensen LT, Jorgensen T, Kirkeby LT, et al. Smoking and alcohol abuse are major risk factors for anastomotic leakage in colorectal surgery. Br J Surg 1999; 86(7):927-31.

24. Nickelsen TN, Jørgensen T, Kronborg O. Lifestyle and 30-day complications to surgery for colorectal cancer, Acta Oncologica. 2005; 44 (3), 218-23.

25. Tùnnesen $H$, Petersen KR, Hùjgaard $L$, et al. Postoperative morbidity among symptom-free alcohol misusers. Lancet. 1992; 340 (8815): 334-37.

26. Tonnesen H, Schutten BT, Jorgensen BB. Influence of alcohol on morbidity after colonic surgery. Dis Colon Rectum. 1987;30(7):549-51.

27. Tonnesen $\mathrm{H}$, Petersen KR, Højgaard L, et al. Postoperative morbidity among symptom-free alcohol misusers. Lancet 1992;340(8815):334-37.

28. Gloria L, Cravo M, Camilo ME, et al. Nutritional deficiencies in chronic alcoholics: relation to dietary intake and alcohol consumption. Am J Gastroenterol 1997; 92 (3): 485-89. 